\title{
2020 CSHP National Awards Program Winners Programme national des prix 2020 de la SCPH : lauréats et lauréates
}

The winner of the Hospital Pharmacy Student Award (co-sponsored by the Canadian Society of Hospital Pharmacists [CSHP] and the Canadian Association of Pharmacy Students and Interns [CAPSI]) is Dillon H Lee (Edmonton, $A B$ ).

Excellence in Pharmacy Practice Interprofessional Collaboration Award Development of Standardized Opioid Prescriptions for Post-Laparoscopic Appendectomy and Cholecystectomy Surgeries and Implementation of Patient Information on Safer Opioid Use (completed at North York General Hospital, Toronto, ON) Jenny C Chiu, Alice Watt
Excellence in Pharmacy Practice - Leadership Award Sponsored by HealthPRO Procurement Services Inc. Development of a Patient-Centered Video Series to Improve Outcomes for Kidney and Lung Transplant Patients (completed at Saskatchewan Transplant Program, Saskatchewan Health Authority, Saskatoon, SK) Holly Mansell, Nicola Rosaasen

\author{
Excellence in Pharmacy Practice - Patient Care Award \\ Sponsored by SteriMax Inc. \\ Radiology Exam and Postoperative/Post-Sedation \\ Breastfeeding: Internal Initiatives and Information \\ Documents (completed at CISSS de l'Outaouais, Gatineau \\ Hospital, Gatineau, QC) \\ Nathalie Gagnon
}

\author{
Development of Standardized Opioid Prescriptions \\ for Post-Laparoscopic Appendectomy and \\ Cholecystectomy Surgeries and Implementation \\ of Patient Information on Safer Opioid Use \\ Excellence in Pharmacy Practice - \\ Inteprofessional Collaboration Award \\ Chiu JC', Srikandarajah $S^{2}$, Smith DE ${ }^{3}$, Watt $A^{4}$, Bishara $T^{1,5}$, \\ Mendez $S^{3}$, Boynton DR \\ ${ }^{1}$ Department of Pharmacy, North York General Hospital, Toronto, Ontario \\ ${ }^{2}$ Department of Anaesthesia, North York General Hospital, Toronto, \\ Ontario \\ ${ }^{3}$ Department of Surgery, North York General Hospital, Toronto, Ontario \\ ${ }^{4}$ Institute for Safe Medication Practices Canada, Toronto, Ontario \\ ${ }^{5}$ Department of Clinical Informatics, North York General Hospital, \\ Toronto, Ontario \\ 'Patient and Family Advisor Program, North York General Hospital, \\ Toronto, Ontario
}

Background: Opioid prescriptions with duration exceeding 7 days for acute pain were associated with double the likelihood of continued use 1 year later. Quantities prescribed vary widely between prescribers. Excess unused opioids are rarely disposed of properly. A 2017 Ontario Student Drug Use Survey showed that 11\% of high school students reported non-medical use of opioids and $55 \%$ of the time they were obtained from home.

Objectives: The objectives were to standardize discharge opioid prescriptions focusing on laparoscopic appendectomy and cholecystectomy (LA \& LC) surgeries and to develop a patient education sheet on opioids.

\begin{abstract}
Methods: A baseline survey was conducted over 3 months in LA/LC patients to establish their opioid usage, pain control, and whether opioid education was received post-operatively. This data was used to develop a standardized prescription. A patient information sheet on proper opioid use, storage and disposal was developed in collaboration with ISMP Canada and support from the Canadian Patient Safety Institute. A post-implementation survey was completed to assess if patients had adequate supply of pain medications and pain control with the new standardized prescription, and to measure rates of opioid education.

Results: Pre-implementation, surgeons prescribed 20 to 30 opioid pills per prescription. The standardized prescription issued 10 tablets. This led to a $56 \%$ decrease in the number of opioids prescribed over the 3-months (from 2672 to 1182 tablets). Results showed that patients were satisfied with their pain control. Patient education on opioids increased from $8.6 \%$ to $44 \%$

Conclusions: Implementing a standardized opioid prescription led to a decrease of 1490 opioid tablets prescribed over 3 months. This would amount to around 11,000 less opioid tablets prescribed over 1 year at 1 institution. The opportunity for other surgical programs and institutions to adopt this prescription would mean several thousand less opioids tablets available for diversion. Increasing patient education may potentially decrease opioid-related misuse.
\end{abstract}

Keywords: opioid, standard prescription, patient education, pain 


\section{Development of a Patient-Centered Video Series to Improve Outcomes for Kidney and Lung Transplant Patients}

\section{Excellence in Pharmacy Practice - Leadership Award Sponsored by HealthPRO Procurement Services Inc.}

Mansell $H^{1}$, Rosaasen $N^{2}$, Mainra $R^{2,3}$, Shoker $A^{2,3}$, Hassan $A^{2,3}$, Trivedi P4, Cardinal L', Fenton ME2,5, Tam F,5

${ }^{1}$ College of Pharmacy and Nutrition, University of Saskatchewan, Saskatoon, SK

${ }^{2}$ Saskatchewan Transplant Program, Saskatchewan Health Authority,

Saskatoon, SK

${ }^{3}$ Division of Nephrology, Department of Medicine, College of Medicine, University of Saskatchewan, Saskatoon, SK

${ }^{4}$ Shoppers Drug Mart, Regina, SK

${ }^{5}$ Division of Respirology, Critical Care and Sleep Medicine, Department of Medicine, College of Medicine, University of Saskatchewan, Saskatoon, SK

Background: Inadequate patient knowledge contributes to poor patient outcomes after transplantation. Kidney and lung transplant patients have indicated that the transplant process is confusing, and they want more education.

Objectives: 1) Conduct a needs assessment to determine optimal format and content, 2) develop a patient-centered educational intervention for kidney patients, 3) test the intervention's effectiveness, 4) develop a similar resource for lung transplant patients.

Methods: Three studies were undertaken providing qualitative and quantitative feedback from patients on the kidney waitlist, kidney transplant recipients, and health care providers working in transplantation. A video series was created for kidney patients by engaging patientstakeholders, experts in medication adherence, video education, motivational psychology, and cultural education. Two randomizedcontrolled trials were designed to test the videos delivered electronically in pre- and post-transplant cohorts. Consultations with patients and caregivers from the lung association informed the content for lung transplant videos.

Results: 'Solid Organ Transplantation: An Educational Mini-Series for Patients' is a 6-part video series outlining the kidney transplant process in its entirety. The videos range between 3 and 24 minutes in length, are patient friendly in design, and incorporate animations to explain complex information to accommodate individuals with low health literacy. Patient testimonials align the content with principles of the adult learning theory. A similarly designed but newly scripted version of the series is intended for the lung transplant audience. Two multicenter, parallel arm, randomized controlled trials are currently being conducted to test the kidney videos (delivered electronically) in pre- and post-transplant cohorts, with collaborators from Saskatoon, Regina, Calgary, Edmonton, Halifax and Chicago.

Conclusion: These studies aim to determine whether electronic education can improve transplant knowledge, patient satisfaction and other patient outcomes (e.g. medication adherence). If proven beneficial, these interventions can be easily implemented and provide consistent, repeatable patient education at low cost, with little impact to existing health care personnel.

Keywords: transplant, video, electronic, education, patient-oriented research, patient perceptions

\section{Radiology Exam and Postoperative/Post-Sedation Breastfeeding: Internal Initiatives and Information Documents}

\section{Excellence in Pharmacy Practice - Patient Care Award Sponsored by SteriMax Inc.}

Gagnon N

Department of Pharmacy, CISSS (Integrated Health and Social Services Centre) de l'Outaouais, Gatineau Hospital, Gatineau, QC

Background: During breastfeeding, tests and procedures requiring contrast media, anesthetics and analgesics may be prescribed. Too often, the tendency is still to tell women they have to pump and throw away their milk, even though this is rarely necessary. In addition, the information being provided is generally inconsistent between departments or healthcare professionals.

Objectives: Review the literature on drugs used in radiology, examination room or operating room in breastfeeding context. Develop a protocol or procedure. Provide written information.

Methods: An Internet search was conducted for such documents, but nothing was found. Phone calls to other CISSS, including those holding a "Baby Friendly Initiative" accreditation, were done; none of them had a protocol or procedure in place. Reference books, literature reviews and specialized databases were consulted.

Results: The use of these drugs in these contexts is a one-time event. They are rapidly eliminated from the plasma compartment, the amount likely to pass into the milk is generally very low with an unlikely digestive absorption in the infant. A resumption of breastfeeding is possible as soon as the patient is in a stable state and has regained a level of alertness authorizing her to stand up.

Conclusions: The procedure "Radiology exam in breastfeeding patient" has been in place since January 2017, while the medical protocol "Postoperative/post-sedation breastfeeding" has been in force since November 2018. The drugs involved are compatible with breastfeeding. The challenge is to widely disseminate these initiatives for the benefit of lactating patients and the children they breastfeed.

Keywords: breastfeeding, drug, contrast agent, radiology, sedation, postoperative 